\title{
Generation of a High-Growth Influenza Vaccine Strain in MDCK Cells for Vaccine Preparedness
}

\author{
Eun-Ha Kim ${ }^{1}$, Hyeok-Il Kwon ${ }^{1}$, Su-Jin Park ${ }^{1}$, Young-Il Kim ${ }^{1}$, Young-Jae Si ${ }^{1}$, In-Won Lee ${ }^{1}$, Se mi Kim ${ }^{1}$, \\ Soo-In Kim², Dong-Ho Ahn', and Young-Ki Choi ${ }^{1 *}$ \\ ${ }^{1}$ Microbiology Department, College of Medicine and Medical Research Institute, Chungbuk National University, Cheongju 28644, Republic \\ of Korea \\ ${ }^{2}$ Virus Vaccine, Green Cross Research Center, Yongin 16924, Republic of Korea
}

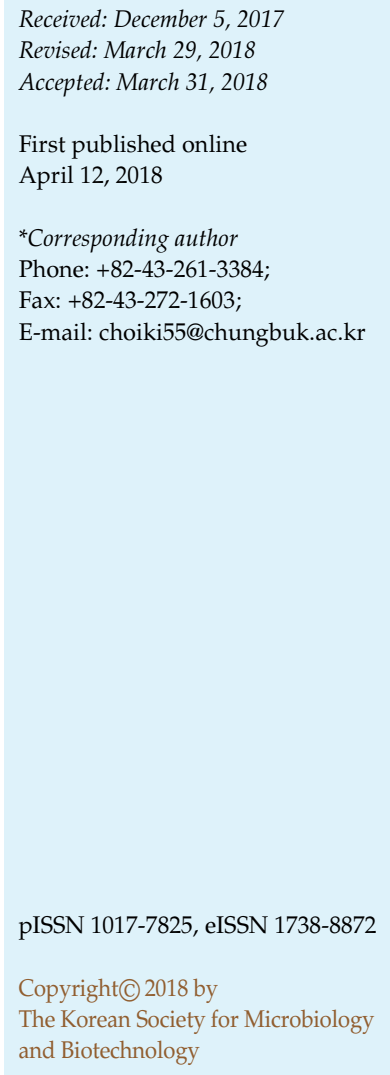

As shown during the 2009 pandemic H1N1 (A(H1N1)pdm09) outbreak, egg-based influenza vaccine production technology is insufficient to meet global demands during an influenza pandemic. Therefore, there is a need to adapt cell culture-derived vaccine technology using suspended cell lines for more rapid and larger-scale vaccine production. In this study, we attempted to generate a high-growth influenza vaccine strain in MDCK cells using an A/ Puerto/8/1934 (H1N1) vaccine seed strain. Following 48 serial passages with four rounds of virus plaque purification in MDCK cells, we were able to select several MDCK-adapted plaques that could grow over $10^{8} \mathrm{PFU} / \mathrm{ml}$. Genetic characterization revealed that these viruses mainly had amino acid substitutions in internal genes and exhibited higher polymerase activities. By using a series of Rg viruses, we demonstrated the essential residues of each gene and identified a set of high-growth strains in MDCK cells $\left(\mathrm{PB} 1_{\mathrm{D} 153 \mathrm{~N}}, \mathrm{M} 1_{\mathrm{A} 137 \mathrm{~T}}\right.$, and $\left.\mathrm{NS} 1_{\mathrm{N} 176 \mathrm{~S}}\right)$. In addition, we confirmed that in the context of the high-growth $\mathrm{A} / \mathrm{PR} / 8 / 34$ backbone, $\mathrm{A} /$ California/7/2009 (H1N1), A/Perth/16/2009 (H3N2), and A/environment/Korea/deltaW150/ 2006 (H5N1) also showed significantly enhanced growth properties (more than $10^{7} \mathrm{PFU} / \mathrm{ml}$ ) in both attached- and suspended-MDCK cells compared with each representative virus and the original PR8 vaccine strain. Taken together, this study demonstrates the feasibility of a cell culture-derived approach to produce seed viruses for influenza vaccines that are cheap and can be grown promptly and vigorously as a substitute for egg-based vaccines. Thus, our results suggest that MDCK cell-based vaccine production is a feasible option for producing large-scale vaccines in case of pandemic outbreaks.

Keywords: Influenza viruses, cell-derived vaccine, Madin-Darby canine kidney (MDCK) cells, bioreactors

\section{Introduction}

Influenza viruses are the major causative agents of respiratory diseases that are responsible for yearly global epidemics infecting millions of people and causing serious illnesses and deaths worldwide [1,2]. The prevention and control of influenza virus infection relies mostly on effective vaccinations. [3]. Conventionally, influenza vaccines are produced using chicken eggs procured from qualified facilities. This method requires millions of eggs, resulting in the need for advanced order prior to production. It takes between 6 and 12 months to produce and distribute the egg-derived vaccines once manufacturing activities have begun. Egg-derived vaccines can also be produced and distributed between 6 and 12 months once the process has started [4]. The disadvantage of the long production time was confirmed during the 2009 H1N1 epidemic when only $22 \%$ of the expected vaccine doses were delivered within the first 6 months after the epidemic was reported $[5,6]$. Moreover, it may not be feasible to manufacture egg-based 
vaccines following pandemics caused by highly pathogenic avian influenza virus (HPAIV), which can kill chickens rapidly. Therefore, the need for an alternative influenza vaccine production method, such as cell-based technology, is apparent [6].

Madin-Darby canine kidney (MDCK) cells are frequently used for the primary isolation of influenza viruses because of their high susceptibility to infection with various influenza strains [7, 8]. Additionally, cell culture-based influenza vaccine production is becoming important as an alternative to egg-based processes [9-11], and MDCK cell culturederived influenza vaccines have been approved by the European Medicines Agency [12] and Korean SK Chemicals [13]. However, the production of influenza vaccines using cell culture has some disadvantages. For example, the volumetric yield of the cell-based flu virus is about 4-fold lower than the egg-based process [14]. This means largevolume bioreactors are required, resulting in high manufacturing costs, which may restrict the use of cell culture-based platforms for influenza vaccine production.

To overcome the current limitations of cell culture-based influenza virus production, we continuously passaged the A/Puerto/8/1934(H1N1) virus (commonly used as the seasonal and pandemic vaccine backbone strain) in MDCK cells to generate an MDCK-adapted high-growth (HG) strain. In addition, throughout the process, we selected several MDCK-adapted PR8 backbone strains by plaque purification. These strains grew up to 10 to 100 times more than the parental strain. Genetic analysis revealed that the selected HG viruses had specific mutations in their internal genes and showed higher polymerase activity compared with the parental virus. Furthermore, in the context of the HG PR8 backbone, various $\mathrm{Rg}$ viruses maintained this high-growth property in both attached and suspended MDCK cells.

\section{Materials and Methods}

\section{Cells}

MDCK (ATCC CCL-34) cells obtained from ATCC were maintained in EMEM (LONZA, Switzerland) using 5\% fetal bovine serum (FBS), 1\% non-essential amino acids (Gibco, USA), and 1\% penicillin/streptomycin (Gibco, USA). Vero cells were cultured in Dulbecco's modified Eagle's MEM (LONZA, Switzerland) with $10 \%$ FBS. Cells were maintained at $37^{\circ} \mathrm{C}$ in $5 \% \mathrm{CO}_{2}$. The MDCK suspension cells were provided by the Green Cross Corporation [15].

\section{Influenza A/Puerto/8/1934 Virus Adaptation in MDCK Cells}

The A/Puerto/8/1934 (PR8, H1N1) virus was propagated in MDCK cells. The PR8 virus was serially passaged 48 times in MDCK cells supplemented with $2 \mu \mathrm{g} / \mathrm{ml}$ TPCK-trypsin (SigmaAldrich, USA) until it acquired the high-growth property $\left(10^{8.0} \mathrm{PFU} / \mathrm{ml}\right)$.

\section{Plasmids and Rescue of Reverse Genetic Viruses}

To generate the HG PR8 backbone virus, each of the eight gene segments of the HG PR8 viruses was amplified by reverse transcription-PCR from plaque-purified viruses and cloned into the vpHW2000 vector, as described in a previous study [16]. All recombinant and point mutation viruses (see Tables 1 and 2) were rescued in Vero and MDCK coculture mixtures (3:1 ratio) as previously described [16]. All rescued viruses were fully sequenced to double check the absence of unwanted mutations.

\section{Site-Directed Mutagenesis}

The individual characteristic mutations in PB2, PB1, PA, M, and NS were introduced into the parental PR8 internal backbone by site-directed mutagenesis using the Gene Tailor site-directed mutagenesis system (Invitrogen, USA) based on the manufacturer's instructions. We resequenced the entire genome to confirm there was no introduction of unwanted mutations.

\section{Growth Curve of Viruses in MDCK Cells}

MDCK cells were inoculated with each mutant virus at a 0.001 MOI (multiplicity of infection), respectively, and incubated at $37^{\circ} \mathrm{C}$ in the appropriate medium containing TPCK-treated trypsin. Virus culture media were harvested at the time points of 12,24 , $36,48,60$, and $72 \mathrm{~h}$ post infection (hpi), and the virus was titrated in MDCK cells by tissue culture infective dose $50\left(\mathrm{TCID}_{50}\right)$. The viral growth property was measured in MDCK cells with three independent trails as previously described [17]. A hemagglutination (HA) test was adapted to determine the virus endpoint titers with

Table 1. Nucleotide and amino acid changes during MDCK adaptation.

\begin{tabular}{|c|c|c|c|c|c|c|c|c|c|c|}
\hline \multirow{2}{*}{$\begin{array}{l}\text { Viral proteins } \\
\text { (position) }\end{array}$} & \multicolumn{2}{|c|}{ P0 } & \multicolumn{2}{|c|}{ P15 } & \multicolumn{2}{|c|}{ P25 } & \multicolumn{2}{|c|}{ P33 } & \multicolumn{2}{|c|}{ P48 } \\
\hline & Nucleotide & Amino acid & Nucleotide & Amino acid & Nucleotide & Amino acid & Nucleotide & Amino acid & Nucleotide & Amino acid \\
\hline PB2 (701) & G & Asp & G & Asp & A & Asn & A & Asn & A & Asn \\
\hline PB1(153) & G & Asp & G & Asp & A & Asn & A & Asn & A & Asn \\
\hline M1(137) & C & Ala & $\mathrm{C}$ & Ala & $\mathrm{C}$ & Ala & A & Thr & A & Thr \\
\hline NS1(176) & A & Asn & A & Asn & G & Ser & G & Ser & G & Ser \\
\hline
\end{tabular}

P0, parent virus A/Puerto/8/1934; P15, 15th MDCK passage; P25, 25th MDCK passage; P33, 33rd MDCK passage; and MA, MDCK-adapted virus after passages. 
Table 2. Growth efficiency of MDCK-adapted high-growth (HG) viruses in MDCK cells.

\begin{tabular}{|c|c|c|c|c|c|c|}
\hline & & & & Virus titer & Plaque size & $\mathrm{MLD}_{50}$ \\
\hline & & & & $\left(\log _{10} \mathrm{PFU} / \mathrm{ml}\right)$ & $(\mathrm{mm})$ & $\left(\log _{10} \mathrm{TCID}_{50} / \mathrm{ml}\right)$ \\
\hline PB2 & PB1 & M1 & NS1 & 6.83 & $1.04 \pm 0.64$ & 3.5 \\
\hline HG-PB2 & HG-PB1 & M1 & NS1 & $8.41^{+}$ & $1.24 \pm 0.24$ & 4.0 \\
\hline HG-PB2 & PB1 & HG-M1 & NS1 & $8.25^{+}$ & $1.12 \pm 0.51$ & 3.5 \\
\hline HG-PB2 & PB1 & M1 & HG-NS1 & $8.4^{+}$ & $1.26 \pm 0.14$ & 3.5 \\
\hline PB2 & HG-PB1 & HG-M1 & NS1 & $8.52^{\ddagger}$ & $1.64 \pm 0.37$ & 3.0 \\
\hline PB2 & HG-PB1 & M1 & HG-NS1 & $8.43^{+}$ & $1.32 \pm 0.12$ & 3.5 \\
\hline PB2 & PB1 & HG-M1 & HG-NS1 & $8.41^{+}$ & $1.19 \pm 0.34$ & 4.0 \\
\hline HG-PB2 & HG-PB1 & HG-M1 & NS1 & $8.53^{\ddagger}$ & $1.74 \pm 0.53$ & 3.5 \\
\hline HG-PB2 & PB1 & HG-M1 & HG-NS1 & $8.17^{+}$ & $1.10 \pm 0.41$ & 4.0 \\
\hline PB2 & HG-PB1 & HG-M1 & HG-NS1 & $8.58^{\ddagger}$ & $1.81 \pm 0.17$ & 3.5 \\
\hline HG-PB2 & HG-PB1 & HG-M1 & HG-NS1 & $8.28^{+}$ & $1.16 \pm 0.11$ & 3.0 \\
\hline
\end{tabular}

${ }^{*} p<0.05$ compared with the parent virus, ${ }^{+} p<0.001$ compared with the parent virus, and ${ }^{\ddagger} p<0.0001$ compared with the parent virus.

$0.5 \%$ turkey erythrocytes [17].

\section{Experimental Infection of Mice}

Groups of 6-week-old female BALB/C mouse $(n=10)$ were intranasally inoculated with each of $10^{2}$ to $10^{7} \mathrm{TCID}_{50} / 50 \mu \mathrm{l}$ of $\mathrm{Rg}$ viruses, which contained double, triple, or quadruplicate amino acid substitutions. Body weights and survival were recorded daily for 14 days.

\section{Virus Propagation in 5-L Single-Use Bioreactors}

MDCK suspension cells were cultured in 5-L single-use bioreactors (PBS Biotech Inc., USA) with a working volume of $3 \mathrm{~L}$, as previously described [18]. When the cell density reached about $1 \times 10^{6}$ cells $/ \mathrm{ml}$, viruses at an MOI of 0.001 and TPCK-trypsin $(5 \mu \mathrm{g} / \mathrm{ml})$ were added to the bioreactors. The virus was yielded at $12,24,36,48,60$, and $72 \mathrm{hpi}$, and was titrated in MDCK cells by $\mathrm{TCID}_{50}[19]$.

\section{Luciferase Minigenome Reporter Assays}

The luciferase reporter plasmids (vpHW72-Luc and canine pHW72-Luc), which contained the Vero cell- and MDCK celldriven polI transcription unit, respectively, were generated in the backbone of vpHW72-Luc, as previously described [17, 20]. Briefly, Vero or MDCK cells were cultured in $24 \mathrm{~h}$ prior to use and $0.1 \mu \mathrm{g}$ each of the vpHW72-Luc or canine pHW72-Luc, vpHW2000-PB2, vpHW2000-PB1, vpHW2000-PA, vpHW2000-NP, and pCMV- $\beta$ galactosidase plasmids were transfected into each cells with TransIT-LT1 transfection reagent, as previously described [16]. Fresh DMEM (Gibco, USA) containing 5\% FBS and incubated at $37^{\circ} \mathrm{C}$ was used to replace the transfection medium after $4 \mathrm{~h}$. Luciferase activity was assayed in triplicate using the luciferase assay system with each of the cell lysates subsequent to washing with PBS after $24 \mathrm{~h}$ (Promega, USA). The results were normalized to the $\beta$-galactosidase activity level of the cells.

\section{Ethics}

General animal care guidelines mandated under the Guidelines for Animal Use and Care of the Korea Center for Disease Control were used for the mouse experimental protocols in this study. The study was approved by the Laboratory and Animal Research Center, under the Institutional Animal Care and Use Committee of Chungbuk National University (Approval No. CBNUA-1052-17-02).

\section{Statistical Analysis}

GraphPad Prism ver. 5.00 for Windows (GraphPad Software, USA) was used to analyze the data. Probability values of less than $0.05(p<0.05)$ were considered statistically significant.

\section{Results}

\section{Growth Efficiency and Genetic Characterization of MDCK-Adapted HG Viruses}

To overcome the relative low growth property of influenza vaccine strains in MDCK cell lines compared with the embryonated chicken egg-derived culture method, we generated an MDCK-adapted A/Puerto/8/1934 vaccine backbone strain by serial passage in MDCK cells. The parental PR8 virus could make plaques after 2 days of infection in MDCK cells (Fig. 1A) and reach peak infectious virus titers of about $6.83 \log _{10} \mathrm{PFU} / \mathrm{ml}$ at $48 \mathrm{~h}$ (Fig. 1B). However, following 48 serial passages including four rounds of plaque purification on the $15^{\text {th }}, 25^{\text {th }}, 33^{\text {rd }}$, and $47^{\text {th }}$ passages in MDCK cells, some of the passaged PR8 purified viruses could make large, clear plaques after 2 days of infection in MDCK cells (Fig. 1A) and reach peak viral titers of $7.5 \log _{10} \mathrm{PFU} / \mathrm{ml}$ (Fig. 1B, a 10-fold increase over parental, $p<0.001$ ). To obtain a single HG PR8 plaque, we 
A
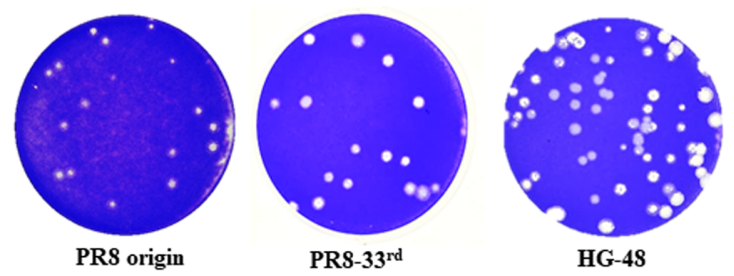

C

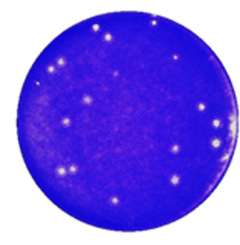

PR8 origin

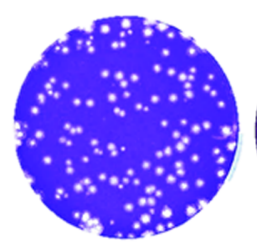

PB2 D701N

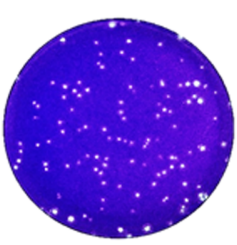

PB1 D153N

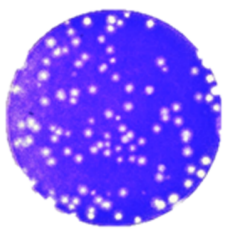

M1 A137T

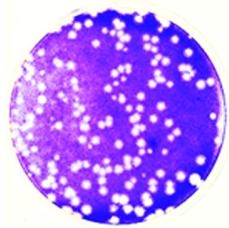

NS1 N176S
B

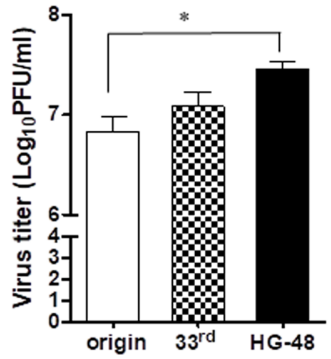

D

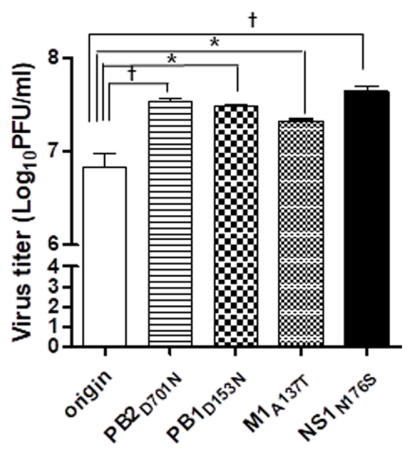

Fig. 1. Growth of MDCK-adapted PR8 and MDCK-adapted high-growth (HG) single-mutant PR8 viruses in MDCK cells.

(A) Plaque morphology of MDCK-adapted HG viruses. (B) Virus titration of MDCK-adapted HG viruses. (C) Plaque morphology of MDCKadapted HG single-mutant PR8 viruses. (D) Virus titration of MDCK-adapted HG single-mutant PR8 viruses. Cells were infected at a multiplicity of infection of $10^{-4}$. Cell culture supernatants were collected at $48 \mathrm{~h}$ post-infection and titered by $\log _{10} \mathrm{PFU} / \mathrm{ml}$. ${ }^{*} p<0.05$ compared with the parent virus, ${ }^{+} p<0.001$ compared with the parent virus. Error bars represent the standard error of the mean. Results were obtained from three independent experiments.

conducted plaque purification again and selected a single clone (HG-48). The large plaque and high-growth properties of the HG-48 clone compared with the parental PR8 virus in MDCK cells were then confirmed (Fig. 1).

To examine when HG-48 acquired the high-growth property, we conducted full-length sequence analysis of the $15^{\text {th }}, 25^{\text {th }}$, and $33^{\text {rd }}$ clones. The first mutations were observed in the PB2 (D701N), PB1 (D153N), and NS1 (N176S) gene segments in the $25^{\text {th }}$ plaque-purified clone (Table 1), and an additional mutation in the M1 gene, M1 (A137T), was observed in the $33^{\text {rd }}$ plaque-purified clone. Furthermore, we found that these amino acid substitutions were stable and maintained for a further 15 passages, giving rise to the HG-48 clone.

To explore the potential role of mutation of each of these four amino acids in the accelerated growth kinetics, we generated mutants of each HG virus using reverse genetics, as previously described [16]. Each HG virus $\left(\mathrm{PB} 2_{\mathrm{D} 701 \mathrm{~N}}\right.$, $\mathrm{PB} 1_{\mathrm{D} 153 \mathrm{~N}}, \mathrm{M} 1_{\mathrm{A} 137 \mathrm{~T}}$, and $\mathrm{NS}_{\mathrm{N} 176 \mathrm{~S}}$ ) was tested for plaqueforming characteristics and growth kinetics in MDCK cells and was compared with the parental PR8 virus (Figs. 1C and 1D). Three HG viruses (PB2 $2_{\mathrm{D} 701 \mathrm{~N}}, \mathrm{M} 1_{\mathrm{A} 137 \mathrm{~T}}, \mathrm{NS}_{\mathrm{N} 176 \mathrm{~S}}$ ) exhibited similar plaque phenotypes (homogeneous) although they were all larger than those of the PR8 parental virus. However, the $\mathrm{PB} 1_{\mathrm{D} 153 \mathrm{~N}}$ virus formed small punctiform plaques with a mean size of $0.45 \pm 0.11 \mathrm{~mm}$ compared with clear circular plaques of $1.04 \pm 0.64 \mathrm{~mm}$ for the PR8 virus (Fig. 1C). As for the growth properties, all four HG viruses $\left(\mathrm{PB} 2_{\mathrm{D} 701 \mathrm{~N}}, \mathrm{~PB} 1_{\mathrm{D} 153 \mathrm{~N}}, \mathrm{M}_{\mathrm{A} 137 \mathrm{~T}}\right.$, and $\mathrm{NS} 1_{\mathrm{N} 176 \mathrm{~S}}$ ) showed significantly increased viral titers (more than 10 times higher) compared with the PR8 parental virus $\left(6.8 \log _{10} \mathrm{PFU} / \mathrm{ml}\right)(p<0.01$, Fig. 1D). Of these, the $\mathrm{NS}_{\mathrm{N} 176 \mathrm{~S}}$ virus showed the highest viral titer of $7.68 \log _{10} \mathrm{PFU} / \mathrm{ml}$ at $48 \mathrm{hpi}$. These results indicate that each substitution within the HG-48 clone contributed to the increased viral titer of this clone in MDCK cells.

\section{MDCK-Adapted HG Viruses Containing a Combination of Mutations Have Enhanced Growth Characteristics}

To determine if there are any synergetic effects of the amino acid substitutions in the PB2, PB1, M1, and NS1 
A

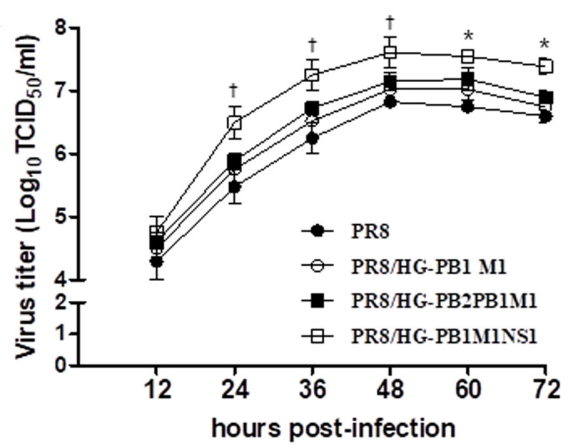

C

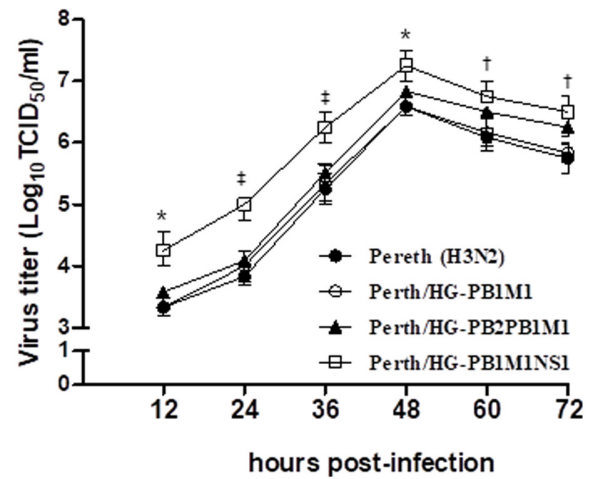

B

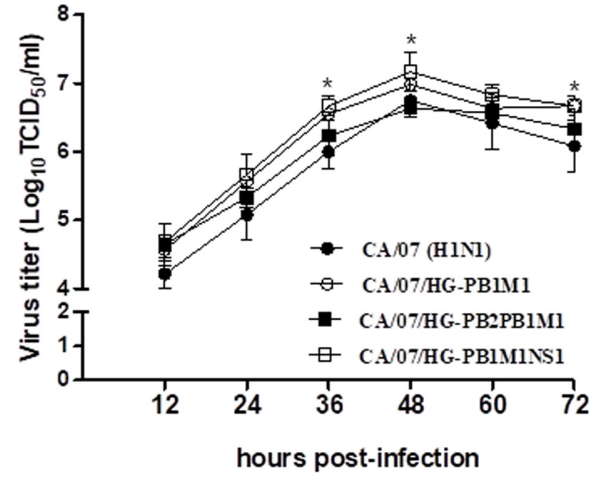

D

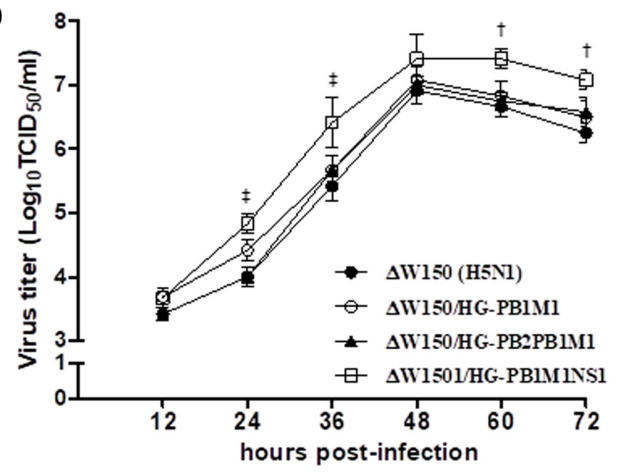

Fig. 2. Growth kinetics of PR8-HG backbone vaccine candidates in MDCK cells.

(A) H1N1 (A/Puerto/8/1934), (B) pH1N1 (A/California/07/2009), (C) H3N2 (A/Perth/16/2009), and (D) H5N1 (A/environment/Korea/ $\Delta \mathrm{W} 150 / 2006)$. Cells were infected at a multiplicity of infection of $10^{-3}$. Cell culture supernatants were collected at $12,24,48,60$, and $72 \mathrm{~h}$ post infection and titered by $\log _{10} \mathrm{TCID}_{50} / \mathrm{ml} .{ }^{*} p<0.05$ compared with the parent virus, ${ }^{+} p<0.001$ compared with the parent virus, and ${ }^{\ddagger} p<0.0001$ compared with the parent virus. Error bars represent the standard error of the mean. Results were obtained from three independent experiments.

segments, we generated various HG viruses with two, three, or four amino acid substitutions and evaluated their replication properties in MDCK cells. We found that three HG viruses (HG-PB1M1, HG-PB2PB1M1, and HG-PB1M1NS1), grew to $8.52,8.53$, and $8.58 \log _{10} \mathrm{PFU} / \mathrm{ml}$ at $48 \mathrm{hpi}$ (Table 2). Strikingly, the HG-PB1M1NS1 combination showed the highest virus titer, thus suggesting that the $\mathrm{PB} 1_{\mathrm{D} 153 \mathrm{~N}}$, $\mathrm{M} 1_{\mathrm{A} 137 \mathrm{~T}}$, and $\mathrm{NS}_{\mathrm{N} 176 \mathrm{~s}}$ substitutions are critical for high growth in MDCK cells. The plaque sizes of each HG virus (HG-PB1M1, HG-PB2PB1M1, and HG-PB1M1NS1) were heterogeneous and larger than the PR8 parental virus, ranging from $\sim 1.64 \mathrm{~mm}$ to $\sim 1.81 \mathrm{~mm}$ during MDCK cell adaptation.

To determine the pathogenicity of the HG viruses in mice, we determined the $50 \%$ mouse lethal dose $\left(\mathrm{MLD}_{50}\right)$ (Table 2). The parental PR8 virus $\mathrm{MLD}_{50}$ was $3.5 \log _{10} \mathrm{TCID}_{50}$, and recombinant $\mathrm{Rg} \mathrm{HG}$ viruses carrying the $\mathrm{PB} 2_{\mathrm{D} 701 \mathrm{~N}}, \mathrm{~PB}_{\mathrm{D} 153 \mathrm{~N}}$, $\mathrm{M1}_{\mathrm{A} 137 \mathrm{~T}}$, or $\mathrm{NS}_{\mathrm{N} 176 \mathrm{~S}}$ gene all showed only moderately attenuated or similar virulence $\left(\mathrm{MLD}_{50}, 3.0-4.0 \log _{10} \mathrm{TCID}_{50}\right)$. These results suggest that the mutant genes of the HG-48 clone do not contribute to increased virulence in mice.

\section{Evaluation of High-Growth Backbone Viruses in Seasonal and HPAI H5N1 Influenza A Strains}

In order to confirm whether the high-growth property of MDCK-adapted HG virus is a general feature, we substituted this backbone (HG) into the recent seasonal human A/H1N1 (A/California/07/2009) and A/H3N2 (A/Perth/16/2009) strains as well as the avian HPAI H5N1 (A/environment/Korea/ $\Delta W 150 / 2006)$ vaccine strain.

First, we tested the one-step growth curves of the parental PR8 and HG viruses (PR8/HG-PB1M1, PR8/HGPB2PB1M1, and PR8/HG-PB1M1NS1) in MDCK cells. As expected, PR8/HG-PB1M1, PR8/HG-PB2PB1M1, and PR8/ HG-PB1M1NS1 showed increased viral titers compared with their parental PR8 virus (48 hpi, 7.0, 7.15, and $7.6 \log _{10} \mathrm{TCID}_{50} / \mathrm{ml}$; Fig. 2A). Moreover, the PR8/HGPB1M1NS1 virus grew more rapidly and exhibited the highest titer in MDCK cells at 24-72 hpi (Fig. 2A).

For the seasonal H1N1 virus study, although similar 
A

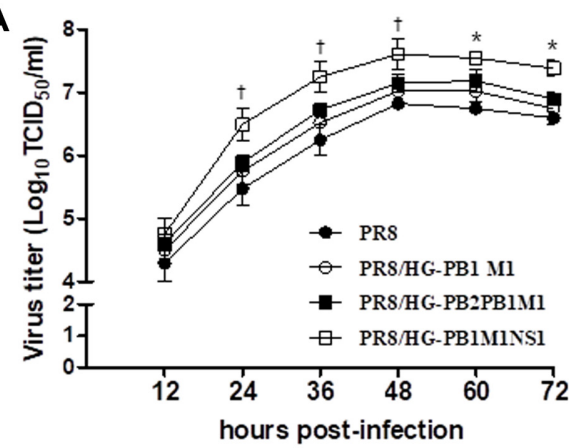

C

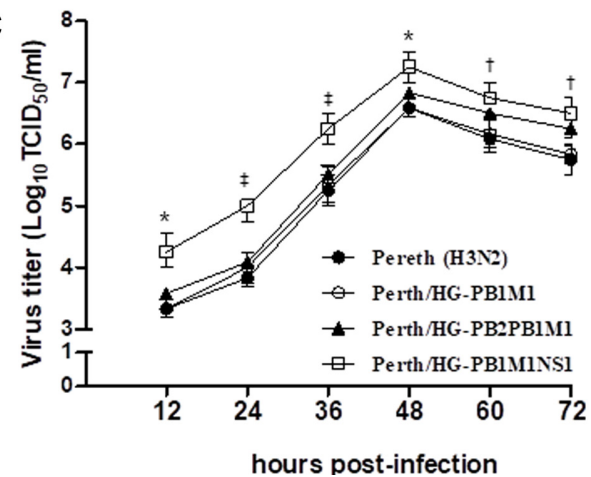

B

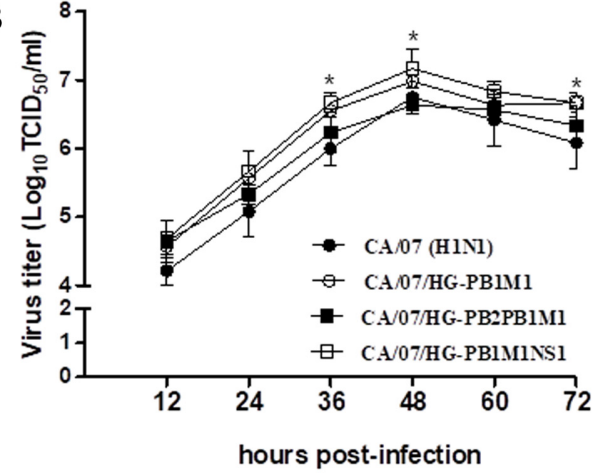

D

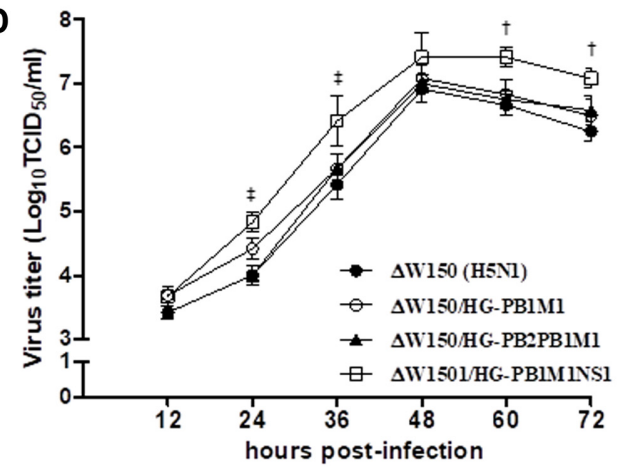

Fig. 3. Growth kinetics of PR8-HG backbone vaccine candidates in MDCK suspension cells.

(A) H1N1 (A/Puerto/8/1934), (B) pH1N1 (A/California/07/2009), (C) H3N2 (A/Perth/16/2009), and (D) H5N1 (A/environment/Korea/ $\Delta \mathrm{W} 150 / 2006)$. MDCK cells were seeded at $5 \times 10^{5}$ cells $/ \mathrm{ml}$ in a $5 \mathrm{~L}$ stirred bioreactor and infected at a multiplicity of infection of $10^{-3}$. Cell culture supernatants were collected at $12,24,48,60$, and $72 \mathrm{~h}$ post-infection and titered by $\log _{10} \mathrm{TCID}_{50} / \mathrm{ml} .{ }^{*} p<0.05$ compared with the parent virus, ${ }^{+} p<0.001$ compared with the parent virus, and ${ }^{\ddagger} p<0.0001$ compared with the parent virus. Error bars represent the standard error of the mean. Results were obtained from three independent experiments.

replication kinetics were observed at early time points (12 and $24 \mathrm{hpi}$ ), the CA/07/HG-PB1M1 and CA/07/HGPB1M1NS1 $(p<0.05)$ viruses showed significantly high titers compared with the parental CA/07 virus at 36,48 , and $72 \mathrm{hpi}$ (Fig. 2B). For the seasonal H3N2 study, the Perth/HG-PB1M1 and Perth/HG-PB2PB1M1 viruses showed similar growth kinetics with the parental A/Perth virus. However, the Perth/HG-PB1M1NS1 virus exhibited significantly higher viral titers (more than 50 -fold increased) at all time points (12-72 hpi) than those of the parental A/ Perth virus $(p<0.05)$ (Fig. $2 \mathrm{C})$, and showed the highest virus titer at $48 \mathrm{hpi}\left(7.25 \log _{10} \mathrm{TCID}_{50} / \mathrm{ml}\right)$. As for the avian H5N1 virus, $\Delta$ W150/HG-PB1M1 and $\Delta$ W150/HG-PB2PB1M1 showed similar growth kinetics to the parental $\mathrm{A} / \Delta \mathrm{W} 150$ virus at all time points. Interestingly, the $\Delta \mathrm{W} 150 / \mathrm{HG}-$ PB1M1NS1 virus exhibited significantly higher growth titers starting at $24 \mathrm{hpi}$ compared with the parental A/ $\Delta \mathrm{W} 150$ virus $(p<0.001)$ and reached a peak viral titer of $7.25 \log _{10} \mathrm{TCID}_{50} / \mathrm{ml}$ at $48-60 \mathrm{hpi}$ (Fig. 2D). These results demonstrate that the HG-PB1M1NS1 combination significantly enhances the replication of pH1N1, H3N2, and H5N1 vaccine strains in MDCK cells.

\section{Application of HG-Backbone Viruses in Large-Scale MDCK Suspension Cultures}

In recent years, the need for development of a serum-free suspension cell culture process for influenza vaccine production has become apparent [15]. To determine which HG virus elicited the greatest increase in vaccine productivity in MDCK suspension cells, each of the HG viruses were inoculated into vaccine-manufacturing MDCK suspension cells (5-L bioreactors) and samples of the culture medium were subjected to the $\mathrm{TCID}_{50}$ assay every $12 \mathrm{~h}$ after virus infection. As shown in Fig. 3A, at $48 \mathrm{hpi}$, the PR8/HG-PB1M1NS1 virus exhibited the highest virus titer of $7.0 \log _{10} \mathrm{TCID}_{50} / \mathrm{ml}$, compared with $6.5 \log _{10} \mathrm{TCID}_{50} / \mathrm{ml}$ for the parental PR8 virus. In contrast, the other HG viruses (PR8/HG-PB1M1 and PR8/HG-PB2PB1M1) did not show 

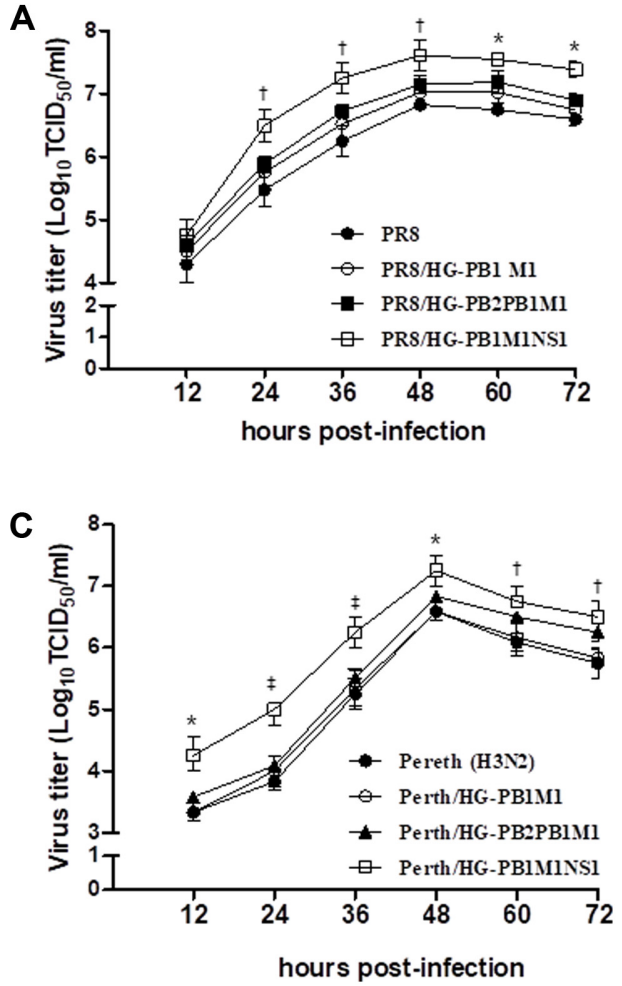
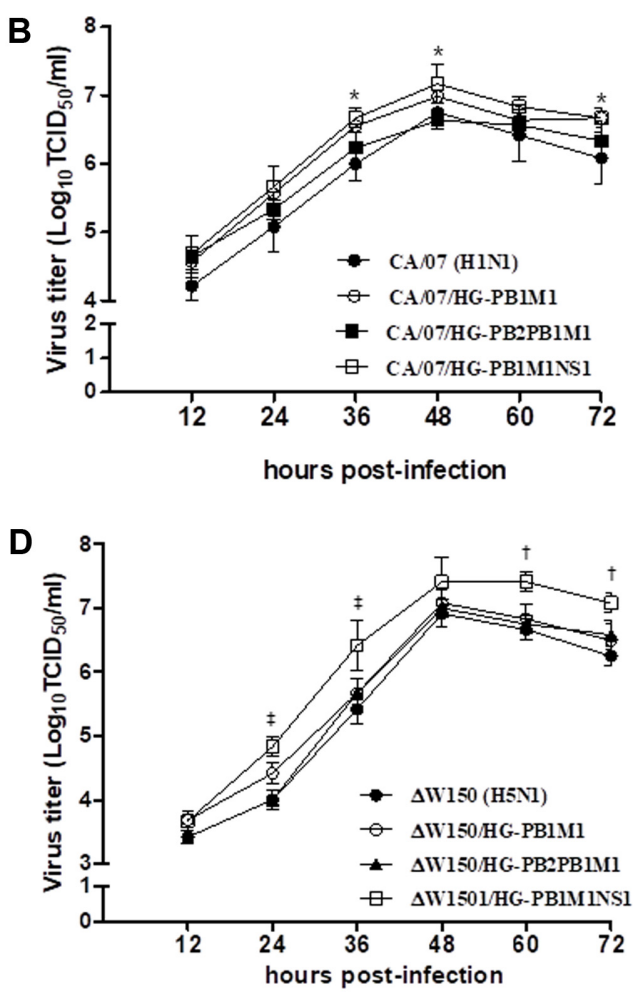

Fig. 4. Enhanced polymerase activity of HG-PR8 backbone viruses.

(A) Vero cells and (B) MDCK cells. Shown are polymerase activities of reconstituted RNP complexes composed of the PB2, PB1, PA, and NP plasmids of parent and HG viruses as well as the indicated combinations and single-point mutants. Note that PA and NP are the same in all complexes, as no mutation was observed in these genes. Luciferase activity values are the means of at least three assays.

significant increases in viral titers over the parental PR8 virus (Fig. 3A).

For the seasonal H1N1 virus, although all HG viruses showed increased virus titers at 24-36 hpi compared with the parental CA/07 virus, CA/07/HG-PB1M1NS1 showed the highest virus titer $\left(7.25 \log _{10} \mathrm{TCID}_{50} / \mathrm{ml}\right)$ at $48 \mathrm{hpi}$ (Fig. 3B). Furthermore, with regard to the seasonal H3N2 virus, Perth/HG-PB1M1NS1 conferred significantly higher titers than the parental Perth virus from early time points $(12-24 \mathrm{hpi}, p<0.001)$ and reached up to $7.25 \log _{10} \mathrm{TCID}_{50} / \mathrm{ml}$ at $48 \mathrm{hpi}$, whereas the other HG backbone viruses (Perth/ HG-PB1M1 and Perth/HG-PB2PB1M1) showed comparable viral growth curves to the parental Perth virus at all time points (Fig. 3C).

It is noteworthy that the avian $\Delta$ W150/HG-PB1M1NS1 virus showed significant high-growth properties compared with the parental $\mathrm{A} / \Delta \mathrm{W} 150$ virus at all the time points except 12 hpi (Fig. 3D). Although the $\Delta$ W150/HG-PB1M1 virus also exhibited high growth at 36 hpi $(p<0.001)$, its growth slowed and eventually reached a similar titer as the parental $\mathrm{A} / \Delta \mathrm{W} 150$ virus $\left(6.5 \log _{10} \mathrm{TCID}_{50} / \mathrm{ml}\right)$. These results clearly demonstrate that the MDCK-optimized HGPB1M1NS1 backbone combination can increase type A influenza virus replication, even in a serum-free large-scale MDCK suspension system.

To determine the pathogenicity of the HG viruses in mice, we determined the $\mathrm{MLD}_{50}$ of each of the Rg H1N1, H3N2, or H5N1 viruses in the HG-PB1M1NS1 backbone. The results showed that the $\mathrm{MLD}_{50}$ of each of the Rg H1N1, H3N2, or H5N1/HG-PB1M1NS1 virus was 3.5, 6.5, and $4.25 \log _{10} \mathrm{TCID}_{50} / \mathrm{ml}$, respectably. These results suggest that the genes of the HG-48 clone do not contribute to increased virulence in mice.

\section{The HG-Backbone Enhances Polymerase Activity}

To investigate the effect of mutations in the polymerase gene of the HG-backbone, we performed a polymerase activity test using a luciferase reporter gene assay in Vero cells. At $37^{\circ} \mathrm{C}$, polymerase complexes containing the individual $\mathrm{PB} 2_{\mathrm{D} 701 \mathrm{~N}}$ and $\mathrm{PB} 1_{\mathrm{D} 153 \mathrm{~N}}$ mutations showed $135 \%$ and $116 \%$ of polymerase activity compared with those of the parent polymerase complex, respectively $(p<0.05$; 
Fig. 4A). It should be noted that the polymerase activity of the double mutant $\left(\mathrm{PB} 2_{\mathrm{D} 701 \mathrm{~N}}\right.$ and $\mathrm{PB} 1_{\mathrm{D} 153 \mathrm{~N}}$ ) was slightly lower $(120 \%)$ than that of $\mathrm{PB} 2_{\mathrm{D} 701 \mathrm{~N}}$ single mutant $(135 \%)$ at $37^{\circ} \mathrm{C}$, but still higher than the parental polymerase complex.

To extend these studies to MDCK cells, we generated a modified luciferase reporter gene system driven by a canine RNA Poll promoter instead of the human RNA PolI promoter and found that the polymerase complexes containing the individual PB2 ${ }_{\mathrm{D} 701 \mathrm{~N}}(188 \%, p<0.001)$ and $\mathrm{PB}_{\text {D153N }}(141 \%, p<0.01)$ mutations showed significantly higher polymerase transcription than that of the parent polymerase complex (Fig. 4B). Coexpression of the PB2 ${ }_{\mathrm{D} 701 \mathrm{~N}}$ mutation with $\mathrm{PB}_{\mathrm{D} 153 \mathrm{~N}}$ also significantly elevated the polymerase activity of the HG virus (150\%, $p<0.01$, Fig. 4B). Thus, these polymerase gene mutations result in increased polymerase activity in MDCK cells.

\section{Discussion}

Mammalian cell culture systems have the potential to be a more robust platform for vaccine generation than the egg production system. The biggest drawback of amplification of influenza viruses in embryonated eggs is the occurrence of antigen variants of parental vaccine strains [20, 21]. In contrast, the HA of influenza viruses amplified using cell culture is typically the same as that of the original strain [22-24]. Considering these advantages, cell culture-based technology has recently been used to manufacture influenza vaccines, some of which are already licensed in Europe [12], Asia [13], and Latin America [25, 26]. However, despite the advantages of cell culture-based influenza vaccine preparation, this method is used in the production of only a small percentage of the influenza vaccines available on the market [27]. This is because cell culture-based vaccines may bring many problems, including higher cost, batch variation, and risk of mycoplasma contamination. To overcome the relatively low viral titer of the cell culturebased method, which leads to the increased vaccine cost compared with egg-based influenza vaccine manufacturing, we adapted the current influenza A vaccine backbone strain A/Puerto/8/1934 (PR8, H1N1) in MDCK cells. Moreover, because recombinant vaccines have proven to be a practical counterplan to traditional influenza vaccines, we selected a high-growth clone, HG-48, that showed a consistently large plaque size and virus titers 10-100 times higher than the parental virus. Interestingly, full-length sequencing showed that the amino acid mutations observed after 48 passages were identical to those at 33 passages, meaning that these mutations were consistently maintained in the HG-48 clone. These data suggest that each of the unique gene substitutions is very stable during MDCK replication (Table 1 ).

When we applied the HG-backbone combination to the recent human seasonal influenza A vaccine strains (H1N1, H3N2, and H5N1), the HG-PB1M1NS1 constellation consistently conferred significantly higher virus titers even at early time points for H1N1 and H3N2. Furthermore, the HG-PB1M1NS1 composition of the HG backbone efficiently enhanced the $\mathrm{H} 5 \mathrm{~N} 1$ virus at all the tested time points. Based on our studies, the $\mathrm{M} 1_{\mathrm{A} 137 \mathrm{~T}}$ and $\mathrm{NS}_{\mathrm{N} 176 \mathrm{~S}}$ mutations together are responsible for the enhanced growth property of candidate vaccine viruses in MDCK cells. Although it is well known that the matrix and NS1 proteins of influenza viruses play important roles for virus growth and interspecies transmission [28, 29], further study is needed to understand the mechanism of action underlying the enhanced growth of viruses with the $\mathrm{M} 1_{\mathrm{A} 137 \mathrm{~T}}$ and $\mathrm{NS} 1_{\mathrm{N} 176 \mathrm{~S}}$ mutations in MDCK cells.

Several studies have reported the profitable development of bioreactor-based suspension cell culture processes using serum-free medium for influenza vaccine production [3033]. Hu et al. [31] reported the feasibility of the development of an MDCK cell-based inactivated $\mathrm{H} 5 \mathrm{~N} 1$ vaccine in a microcarrier-based bioreactor culture system. To this end, they used a $2 \mathrm{~L}$ bioreactor ( $1 \mathrm{~L}$ working volume) with peak titers reaching $10^{8-9} \mathrm{TCID}_{50} / \mathrm{ml}$. In 2007, the European Medicines Agency approved an MDCK cell culture-derived influenza vaccine (CCIV) (Optaflu; Novartis Vaccines) produced using bioreactors of more than 100 L [11]. To evaluate virus productivity in MDCK suspension cells, each of the HG-backbone viruses were inoculated into vaccine-manufacturing, serum-free MDCK suspension cells as 5-L large-scale preparations in bioreactors and virus replication was compared. Interestingly, all H1N1, H3N2, and avian $\mathrm{H} 5 \mathrm{~N} 1$ viruses containing the HG-PB1M1NS1 backbone consistently exhibited high viral titers, with the avian H5N1 virus showing significantly enhanced virus productivity (up to 100 times greater than parental). Therefore, our results could help establish MDCK-optimized HG-backbone large-scale vaccine production.

There are many factors that contribute to viral growth in vitro, such as cell line passage number, TPCK-trypsin concentration, media brands, and temperature. However, our selected backbone strains consistently showed significantly high virus titers and polymerase activities compared with those of the original PR8 backbone virus (three independent trials). Therefore, we believe that the selected PR8 backbone viruses have increased proliferation 
ability in MDCK cells.

Several studies have suggested that a second booster vaccine or increased antigenic dose is required in very young or elderly patients to induce proper protective immunity, since their immune status is immature or attenuated [34-36]. Furthermore, avian H5N1 mock-up vaccines have shown relatively low immunogenicity in mammalian hosts, which is daunting in terms of avian influenza vaccine preparedness $[36,37]$. Hence, some studies have suggested increasing antigen doses to as much as $15 \mu \mathrm{g} /$ dose to induce proper immunogenicity [38]. In light of this, production using our MDCK-adapted HG-PB1M1NS1 backbone-based vaccine in serum-free MDCK suspension cells could be a rapid and useful strategy to meet the need for high-dose HPAI vaccine preparedness.

The MDCK-adapted virus in this study exhibited stable mutations in both the PB2 and PB1 viral proteins, which are components of the RNA-dependent polymerase complex. Therefore, we first performed a luciferase reporter gene assay in Vero cells to investigate whether $\mathrm{PB} 2_{\mathrm{D} 701 \mathrm{~N}}$ and $P B 1_{D 153 N}$ mutations affect polymerase activity. The PB2 ${ }_{D 701 N}$ and $P B 1_{D 153 \mathrm{~N}}$ mutations elevated the polymerase activity of HG viruses, resulting in substantially higher transcription/ replication activity. MDCK cells have recently been approved for human vaccine production. Therefore, to further compare the polymerase activity of the MDCK cellspecific PolI promoter with that of the original human Poll promoter system, we assessed the polymerase activity, as described previously [39]. These results showed that $P B 2_{D 701 N}$ and $P B 1_{D 153 N}$ mutations found in the $H G$ virus confer an increase in polymerase activity over that of the parental virus. The PB2 polymerase gene is known to be associated with polymerase activity, virulence, and restriction of host range. [40]. For this reason, we checked the virulence of PB2 mutants in mice by assessing the $\mathrm{MLD}_{50}$. Unexpectedly, the $H G$ viruses showed similar $L_{50}$ values as the PR8 virus (Table 2). Moreover, the HG backbone did not increase virulence when introduced into H1N1, H3N2, or H5N1 viruses. Thus, the HG-backbone viruses may be useful to develop safer and efficacious vaccines.

The production of a capable system to supply influenza vaccine to the world while keeping safety, immediacy, and cost-effectiveness in mind remains an important challenge for international government agencies. Taken together, our results show that the HG-backbone vaccine viruses identified in this study can improve the titers of seasonal and pandemic influenza vaccines in cultured cells. Thus, future efforts to further explore the feasibility of this method for vaccine production is warranted.

\section{Acknowledgments}

This research was supported by the Korea Healthcare Technology R\&D Project funded by the Ministry of Health and the Korea National Research Institute of Health, Republic of Korea (Grant No. A103001 and HI16C1032).

\section{Conflict of Interest}

The authors have no financial conflicts of interest to declare.

\section{References}

1. Garten RJ, Davis CT, Russell CA, Shu B, Lindstrom S, Balish A, et al. 2009. Antigenic and genetic characteristics of swineorigin 2009 A (H1N1) influenza viruses circulating in humans. Science 325: 197-201.

2. Saito T, Nakaya $Y$, Suzuki $T$, Ito $R$, Saito $T$, Saito $H$, et al. 2004. Antigenic alteration of influenza $B$ virus associated with loss of a glycosylation site due to host-cell adaptation. J. Med. Virol. 74: 336-343.

3. Grohskopf LA, Olson SJ, Sokolow LZ, Bresee JS, Cox N, Broder KR, et al. 2016. Prevention and control of seasonal influenza with vaccines: recommendations of the advisory committee on immunization practices (ACIP)-United States, 2016-17 influenza season. MMWR Recomm. Rep. 65: 1-54.

4. Dawood FS, Iuliano AD, Reed C, Meltzer MI, Shay DK, Cheng P-Y, et al. 2012. Estimated global mortality associated with the first 12 months of 2009 pandemic influenza A H1N1 virus circulation: a modelling study. Lancet Infect Dis. 12: 687-695.

5. Larson RC, Teytelman A. 2012. Modeling the effects of H1N1 influenza vaccine distribution in the United States. Value Health 15: 158-166.

6. Lee M-S, Hu AY-C. 2012. A cell-based backup to speed up pandemic influenza vaccine production. Trends Microbiol. 20: 103-105.

7. Gaush CR, Smith TF. 1968. Replication and plaque assay of influenza virus in an established line of canine kidney cells. J. Appl. Microbiol. 16: 588-594.

8. Govorkova E, Matrosovich M, Tuzikov A, Bovin N, Gerdil C, Fanget B, et al. 1999. Selection of receptor-binding variants of human influenza A and B viruses in baby hamster kidney cells. Virology 262: 31-38.

9. Audsley JM, Tannock GA. 2008. Cell-based influenza vaccines. Drugs 68: 1483-1491.

10. Genzel Y, Reichl U. 2009. Continuous cell lines as a production system for influenza vaccines. Expert Rev. Vaccines 8: 1681-1692.

11. Ulmer JB, Valley U, Rappuoli R. 2006. Vaccine manufacturing: challenges and solutions. Nat. Biotechnol. 24: 1377-1383.

12. Doroshenko A, Halperin SA. 2009. Trivalent MDCK cell 
culture-derived influenza vaccine Optaflu (Novartis Vaccines). Expert Rev. Vaccines 8: 679-688.

13. Song JY, Cheong HJ, Lee J, Woo HJ, Wie S-H, Lee J-S, et al. 2015. Immunogenicity and safety of a cell culture-derived inactivated trivalent influenza vaccine (NBP607): a randomized, double-blind, multi-center, phase 3 clinical trial. Vaccine 33: 5437-5444.

14. HSA. 2014. Understanding vaccines, vaccine development and production. Available from http://www.hsa.gov.sg/ content/hsa/en/Health_Products_Regulation/Consumer_ Information/Public_Advisories/Influenza_A_H1N1_information/ H1N1_Vaccines/understanding-vaccines--vaccine-developmentand-production.html. Accessed Sep. 24, 2012.

15. Shin D, Park KJ, Lee H, Cho EY, Kim MS, Hwang MH, et al. 2015. Comparison of immunogenicity of cell- and egg-passaged viruses for manufacturing MDCK cell culture-based influenza vaccines. Virus Res. 204: 40-46.

16. Song M-S, Baek YH, Pascua PNQ, Kwon H-I, Park S-J, Kim E-H, et al. 2013. Establishment of Vero cell RNA polymerase I-driven reverse genetics for influenza A virus and its application for pandemic (H1N1) 2009 influenza virus vaccine production. J. Gen. Virol. 94: 1230-1235.

17. Song M-S, Pascua PNQ, Lee JH, Baek YH, Lee O-J, Kim C-J, et al. 2009. The polymerase acidic protein gene of influenza A virus contributes to pathogenicity in a mouse model. $J$. Virol. 83: 12325-12335.

18. Lohr V, Genzel Y, Behrendt I, Scharfenberg K, Reichl U. 2010. A new MDCK suspension line cultivated in a fully defined medium in stirred-tank and wave bioreactor. Vaccine 28: 6256-6264.

19. Reed LJ, Muench H. 1938. A simple method of estimating fifty per cent endpoints. Am. J. Epidemiol. 27: 493-497.

20. Rocha EP, Xu X, Hall HE, Allen JR, Regnery HL, Cox NJ. 1993. Comparison of 10 influenza A (H1N1 and H3N2) haemagglutinin sequences obtained directly from clinical specimens to those of MDCK cell- and egg-grown viruses. J. Gen. Virol. 74: 2513-2518.

21. Schild G, Oxford J, De Jong J, Webster R. 1983. Evidence for host-cell selection of influenza virus antigenic variants. Nature 303: 706-709.

22. Dumitrescu M, Grobnicu M, Zilişteanu E, Alexandrescu V. 1981. A three years experience in using MDCK cell line for influenza virus isolation (1979-1981). Arch. Roum. Pathol. Exp. Microbiol. 40: 313.

23. Gambaryan AS, Karasin AI, Tuzikov AB, Chinarev AA, Pazynina GV, Bovin NV, et al. 2005. Receptor-binding properties of swine influenza viruses isolated and propagated in MDCK cells. Virus Res. 114: 15-22.

24. Stevens J, Chen L-M, Carney PJ, Garten R, Foust A, Le J, et al. 2010. Receptor specificity of influenza A H3N2 viruses isolated in mammalian cells and embryonated chicken eggs. J. Virol. 84: 8287-8299.

25. Pradesh A. 2012. Novartis receives FDA approval for
Flucelvax, the first cell-culture vaccine in US to help protect against seasonal influenza. Available from http://www. andhranews.net/Business/2012/Novartis-receives-FDA-approvalFlucelvax-first-53291.htm. Accessed Nov. 20, 2012.

26. Tanzi MG. 2013. Flucelvax: first seasonal vaccine using cellculture technology. Pharm. Today 19: 31.

27. Ping J, Lopes TJ, Nidom CA, Ghedin E, Macken CA, Fitch A, et al. 2015. Development of high-yield influenza A virus vaccine viruses. Nat. Commun. 2: 8148.

28. Hale BG, Randall RE, Ortín J, Jackson D. 2008. The multifunctional NS1 protein of influenza A viruses. J. Gen. Virol. 89: 2359-2376.

29. Watanabe T, Imai M, Kawaoka Y. 2017. NS1 is the fluid for "flu-transmission". Proc. Natl. Acad. Sci. USA 2017 114: 11012-11014.

30. Genzel Y, Olmer R, Schäfer B, Reichl U. 2006. Wave microcarrier cultivation of MDCK cells for influenza virus production in serum containing and serum-free media. Vaccine 24: 6074-6087.

31. Hu AYC, Weng TC, Tseng YF, Chen YS, Wu CH, Hsiao S, et al. 2008. Microcarrier-based MDCK cell culture system for the production of influenza $\mathrm{H} 5 \mathrm{~N} 1$ vaccines. Vaccine 26: 5736-5740.

32. Merten O, Manuguerra J, Hannoun C. 1999. Production of influenza virus in serum-free mammalian cell cultures. Dev. Biol. Stand. 98: 73-24.

33. Tree JA, Richardson C, Fooks AR, Clegg JC, Looby D. 2001. Comparison of large-scale mammalian cell culture systems with egg culture for the production of influenza virus A vaccine strains. Vaccine 19: 3444-3450.

34. Hannoun C. 2013. The evolving history of influenza viruses and influenza vaccines. Expert Rev. Vaccines 12: 1085-1094.

35. Keitel W, Groth N, Lattanzi M, Praus M, Hilbert AK, Borkowski A, et al. 2010. Dose ranging of adjuvant and antigen in a cell culture $\mathrm{H} 5 \mathrm{~N} 1$ influenza vaccine: safety and immunogenicity of a phase 1/2 clinical trial. Vaccine 28: 840-848.

36. Qiao C, Tian G, Jiang Y, Li Y, Shi J, Yu K, et al. 2006. Vaccines developed for $\mathrm{H} 5$ highly pathogenic avian influenza in China. Ann. N.Y. Acad. Sci. 1081: 182-192.

37. Shi H, Liu XF, Zhang X, Chen S, Sun L, Lu J. 2007. Generation of an attenuated H5N1 avian influenza virus vaccine with all eight genes from avian viruses. Vaccine 25: 7379-7384.

38. Roy P, Noad R. 2009. Virus-like particles as a vaccine delivery system: myths and facts. Adv. Exp. Med. Biol. 655: 145158.

39. Murakami S, Horimoto T, Yamada S, Kakugawa S, Goto H, Kawaoka Y. 2008. Establishment of canine RNA polymerase I-driven reverse genetics for influenza A virus: its application for H5N1 vaccine production. J. Virol. 82: 1605-1609.

40. Gabriel G, Abram M, Keiner B, Wagner R, Klenk H-D, Stech J. 2007. Differential polymerase activity in avian and mammalian cells determines host range of influenza virus. J. Virol. 81: 9601-9604. 